\title{
In vivo identification of soft biological tissues using MR imaging
}

\author{
Laura Bouten*,*** — Sylvain Drapier**,*** \\ * Biomaterials and Mechanics Dept. - Engineering and Health Division \\ ** Mechanics and Materials Processing Dept. - Struc. and Mat. Science Division \\ *** LTDS - UMR CNRS 5513 \\ Ecole Nationale Supérieure des Mines de Saint-Etienne \\ 158 , cours Fauriel \\ F-42023 Saint-Etienne cedex 2 \\ \{bouten,drapier\}@emse.fr
}

\begin{abstract}
Soft biological tissues constituting the human body are individually investigated regarding the identification of their mechanical properties. This study proposes an identification process of human legs tissues by coupling Magnetic Resonance Imaging (MRI) images with different levels of stocking used as mechanical loading. This loading may be compared with a compression around the whole leg section. The identification method relies on one hand on MRI image processing (digital image correlation, volume extraction, etc.) and on the other hand on a Finite Element Model combined with an optimization algorithm.

RÉSUMÉ. Les tissus biologiques mous constituant le corps humain font individuellement l'objet d'études visant à déterminer leurs propriétés mécaniques. L'étude propose l'identification des propriétés mécaniques des tissus de la jambe par le couplage d'images par résonance magnétique (IRM) et de différents niveaux de contention comme chargement mécanique. Celui-ci peut être assimilé à une compression sur une section complète de jambe. La méthode d'identification fait intervenir d'une part, le traitement des images IRM (corrélation d'images numériques, extraction des volumes, etc.) et, d'autre part, la modélisation par la méthode aux éléments finis combinée à un algorithme d'optimisation.

KEYWORDS: identification, MRI, stocking, finite element, optimization.

MOTS-CLÉS : identification, IRM, contention, éléments finis, optimisation.
\end{abstract}

DOI:10.3166/EJCM.18.21-32 @ 2009 Lavoisier, Paris

EJCM - 18/2009. Numerical models in biomechanics, pages 21 to 32 


\section{Introduction}

Medicine is more and more attentive to engineering techniques to help medical practitioners in diagnosing, observing, and understanding harms. Biomechanics is one privileged field where biology and engineering yield together responses to medicine. The present study is a part of a long term approach which will help angiologists in vascular system deficiencies healing, and also to assess veinous treatments efficiency by combining medical imaging and numerical physical models. Leg problems are widespread throughout the world and most of them originate within the veins. One way to face chronic veinous deficiency is to wear stockings or compression socks. The efficiency of the garments on the superficial veins is known and they are mostly used for avoiding pathology such as phlebitis, lower limb ulcers and for facilitating the veinous return. After some clinical observations, stockings are supposed to have an important effect on the deep veinous system. The main aim of our study is to identify the material parameters of the soft biological tissues constituting the leg, in order to predict how the compression will act on the deep veins. This study uses the compression of the stockings as a mechanical loading to perform tests which are necessary to carry out a numerical identification process. More generally, the study presented here is the first application of a non-invasive and non-traumatic identification methodology in vivo.

The presented identification approach consists in coupling MRI (Magnetic Resonance Imaging) images with FEM (Finite Element Modelling). On the one hand, the digital image correlation technique is used to get experimental data and on the other hand, the 'ZeBuLoN' code is used to compute the Finite Element Analyses. This methodology could be, in the future, applied on every type of limb morphology. Behind the medical aspect and its application by phlebologists in medical prescription of stockings, our principal interest and what makes the originality of the study is the coupling of MRI images with compression socks as mechanical loading. The methodology and its setting up will be presented first, along with the tools used and then, the first results and observations will be discussed.

\section{Methods}

\subsection{In vivo experimental test and data}

The data acquisition stage is based on MRI. It is applied on a 25 years old woman who does not present any veinous pathology. As the first aim of the study is to develop a methodology, the tests are performed on only one person for the time being. These tests consists in wearing successively two different compression socks, one yielding a pressure of $20 \mathrm{mmHg}(2.7 \mathrm{kPa})$ and the other one, a pressure of $40 \mathrm{mmHg}(5.3 \mathrm{kPa})$. The announced pressure is an approximation but can easily be calculated from local radii of curvature of the leg (Gaied et al., 2006). With each sock put on, the patient enters a MRI device where 2 sets of 10 images are taken. Slices are acquired sequentially 
with a $5 \mathrm{~mm}$ thickness and the pixel size is about $(0.36 * 0.36) \mathrm{mm}^{2}$. The first set is 10 T1-Weighted images along $5 \mathrm{~cm}$ of the calf and the second one is T2-Weighted with fat saturation. T1-Weighted images emphasize the contrast between muscles, bones and adipose and are obtained under a spin echo sequence with a repetition time (TR) of $772 \mathrm{~ms}$ and a echo time (TE) of $13 \mathrm{~ms}$. As the vascular system is our principal interest, T2-Weighted images are also needed. They are obtained with a TR of 426 $\mathrm{ms}$ and a TE of $24 \mathrm{~ms}$. Moreover, to get more contrasted T2-Weighted images a radio contrast medium could be injected in the calf but then the methodology developed here would lose its non-invasive feature.

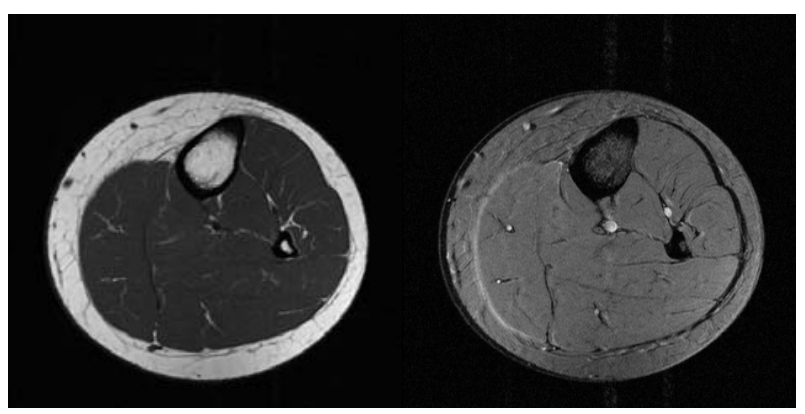

Figure 1. T1-weighted (left) and T2-weighted (right) images with a compression of $40 \mathrm{mmHg}$ taken at mid-calf.

\subsection{Correlation process}

The digital image correlation process is used for getting displacement data in characteristic areas of the calf. The correlation process consists in comparing pairs of images of the same phenomenon but in various states of transformation. Each pair of images is partitioned with a set of windows called correlation windows. These ones are compared two by two i.e. a correlation window and its associate in the second image. The correlation peak is defined as the maximum of similarity, in a mathematical sense (Ducottet et al., 1994), of the two compared windows. Results are displacements needed for the centre of the correlation windows to reach their correlation peak.

\subsection{Geometry reconstruction}

In identification process, experimental data are used along with simulated data of the same phenomenon which are obtained in our methodology by a Finite Element Model (FEM). Then, a realistic mesh of the considered leg section is required. To reconstruct the whole calf geometry considering its different muscles, a modelling software with a high precision of segmenting process must be used. The Amira ( software is chosen. Bones and adipose are automatically detected by the segmentation 
process. The muscle regions presenting low contrasts are delineated with the same software but manually. Once delineated, segmentation results are converted into a 2D-mesh.

\subsection{Constitutive model}

According to previous studies (Fung, 1993, Bosboom et al., 2001), most of soft biological tissues are hyperelastic, incompressible and ideally viscoelastic. In order to fit the biological (physiological) response, hyperelastic models are used without viscosity that can not be accounted for in our process due to the MRI acquisition which is quite long in time and hence will "average" the response over few minutes.

A three-term Mooney-Rivlin strain energy formulation is used to simulate the fatskin complex response and a two-term one is used to simulate both vascular system and muscles response. The Mooney-Rivlin model can be written in the strictly incompressible case as,

$$
W\left(I_{1}, I_{2}\right)=\sum_{i, j=0}^{\infty} C_{i j}\left(I_{1}-3\right)^{i}\left(I_{2}-3\right)^{j}
$$

with $I_{1}$ and $I_{2}$ the first and second invariants of the right Cauchy-Green strain tensor. A previous study (Drapier et al., 2007) has shown that the socks had, in threedimension, a hypoelastic behavior, but the model was simplified here, and a classical Mooney-Rivlin (two-term formulation) constitutive law was used. Each material in the model is assumed hyperelastic and almost or totally incompressible. This choice leads to numerical difficulties, but the assumption of incompressible material is required to take into account the fact that biological tissues are widely composed of water. For justifying how a model is written, one can refer to the molecular theory of hyperelasticity (Ogden, 1982).

\subsection{Numerical model}

The model proposed in the present study is a plane strain model with totally or almost incompressible Mooney-Rivlin material constitutive laws. It has been solved through a finite element approach using the ZeBuLoN code. According to the constitutive laws chosen, the use of mixed elements is mandatory. Indeed, displacement-based finite element method is not able to predict properly stresses within a totally incompressible material and has difficulties with the analysis of almost incompressible material (ill-conditioning of the stiffness matrix, 'locking', spurious stresses... ). A way to encompass these difficulties is to use a $u / p$ formulation (Weiss, 1994), i.e. elements are formulated in displacement and pressure. The quasi-incompressibility is enforced here using Lagrange multipliers (Sussman et al., 1987). Finite strains are also used because this problem is then strongly nonlinear. 
Furthermore, another nonlinearity is added, since the simulation should include the stocking response which involves a large contact area between the garment and the skin. As the skin is assumed not perfectly smooth, a friction coefficient is introduced, which helps also to guarantee the unicity of the solution. A penalty method is chosen to solve the contact problem. As the incompressibility is solved by Lagrange multipliers, it is reasonable to use a penalty method instead of further Lagrange multipliers which would introduce another set of unknowns. Indeed, if the number of Lagrange multipliers is greater than kinematic unknowns, the problem is not solvable.

The mechanical loading is a compression around the whole leg, applied by the stretched sock. Therefore, the sock must be put in tension first (Figure 2(a)), starting from an initial unloaded state. The second step consists in releasing the applied internal pressure (Figure 2(b)) in the sock, in order to come in contact with the leg and to apply the corresponding stocking pressure (Figure 2(c)). In the schematics (Figure 2), dotted lines are the defined contact areas which do not exist in the first step of the computation (swelling).

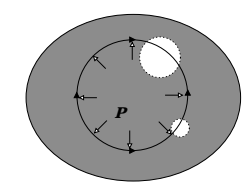

(a)

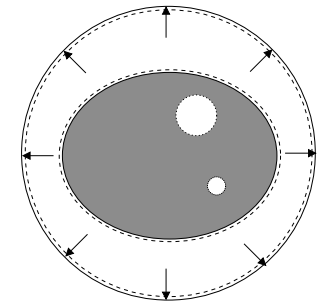

(b)

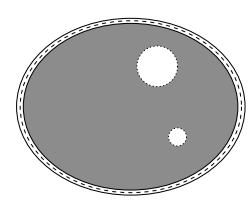

(c)

Figure 2. Schematics of the three steps computations: (a) initial state, (b) garment put in tension, (c) garment comes in contact with the skin after "unswelling" ( - - contact zones).

\subsection{Identification process}

Experimental data and simulated ones are compared in the identification process, so as to determine material parameters which will lead to the best approximation of the experiments .

The identification process is then performed by the minimization of an objective function [2] which expresses the distance between simulated and experimental displacements. The objective function is written as,

$$
\mathcal{F}(\theta)=\frac{1}{2 N} \sum_{i=1}^{N}\left(u\left(P_{i}\right)^{e}-u\left(P_{i}, \theta\right)^{s}\right)^{2}
$$

where $\theta$ is the vector of the unknown material parameters, $N$ the number of geometrical points, $u\left(P_{i}\right)^{e}$ experimental displacements at the $i$ th point and $u\left(P_{i}, \theta\right)^{s}$ 
theoretical (simulated) displacements expressed at geometrical point $P_{i}$. Let us note that the displacements used for this distance must coincide with the same point, both in experiments and computation. It was chosen to measure these displacements at the geometrical points corresponding to the center of digital image correlation windows.

At the moment, the algorithm employed to solve this nonlinear minimization problem has not been firmly selected. Levenberg-Marquardt method is widely used identification problems. As the aim is also to save time, another method should be tested such as the Nelder-Mead method (Luersen et al., 2004). These methods must be compared in order to determine which one is the more efficient in our case.

\section{Results}

\subsection{Correlation results}

The correlation process is performed on MR images. As explained before, only two images are needed to obtain correlation results. The two images chosen, in the same planigraphic plane, are situated where the calf is the largest, i.e. at approximatively $300 \mathrm{~mm}$ from the ankle. One image is extracted from the set of $40 \mathrm{mmHg}$ pressure compression test and the other one from the set of $20 \mathrm{mmHg}$ pressure compression. After overlaying these two images, one can see that a rigid body motion exists between the two states of loading It is chosen to align these images by performing a rotation and a translation, so that the surface center of the bones coincide in both images. The next step of the process is to define correlation windows. After few tests with different sizes of windows, these chosen are $64 \times 64$ pixels and $16 \times 16$ pixels with a searching area 4 times greater than the correlation windows. Windows must be placed on the geometry, and the most relevant way is to put them on regions of interest, which are mainly aponeuroses, skin and vascular regions. The windows are approximatively 160 in number. Results are displacements in pixels of the correlation peak of each window (Figure 3). The correlation results presented here are results of both set of images, T1-weighted and T2-weighted. As a matter of fact, getting correlation results over the vascular zones is realized by using T2-weighted images whereas T1-weighted images are used for the other tissues.

\subsection{Segmentation and meshing results}

Since a significant contrast is needed, only T1-weighted images are used for segmentation. Taking into account the contrast in the image, a first threshold has been defined in order to separate adipose from other tissues. In T1-weighted images adipose is light-coloured. Contours are defined manually following the aponeuroses, because the contrast between the different muscles is not sufficient. The segmenting process must be done on two planes to obtain a 3D-mesh. To encompass this restriction, it has been done on two similar images. The obtained mesh is not totally satisfactory 


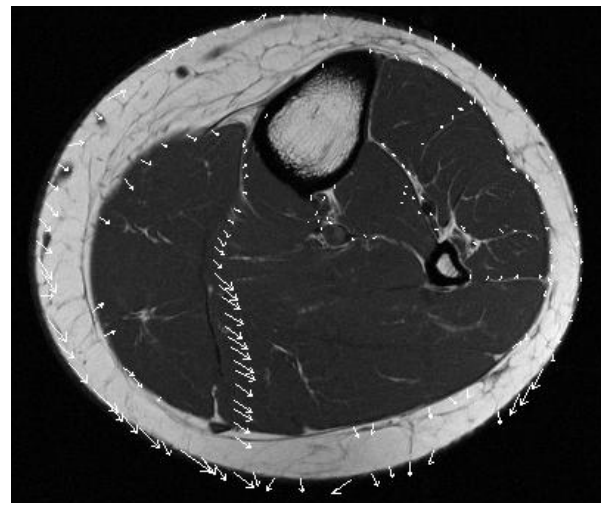

Figure 3. Digital image correlation results between a $40 \mathrm{mmHg}$ and $20 \mathrm{mmHg}$ pressure compression. (minimal displacement : $0.035 \mathrm{~mm}$ and maximal displacement: $8.9 \mathrm{~mm})$

because a 2D-mesh is needed for the finite element model. So, a new functionality is written in $\mathrm{ZeBuLoN}$ to convert $3 \mathrm{D}$ tetrahedral mesh into $2 \mathrm{D}$ triangle mesh. The geometry is reconstructed by Amira $\odot$ and is smoothed and its accuracy is probably sufficient (Figure 4). The obtained mesh is made up of 5461 elements and the number of nodes depends on the type of element used (linear or quadratic).

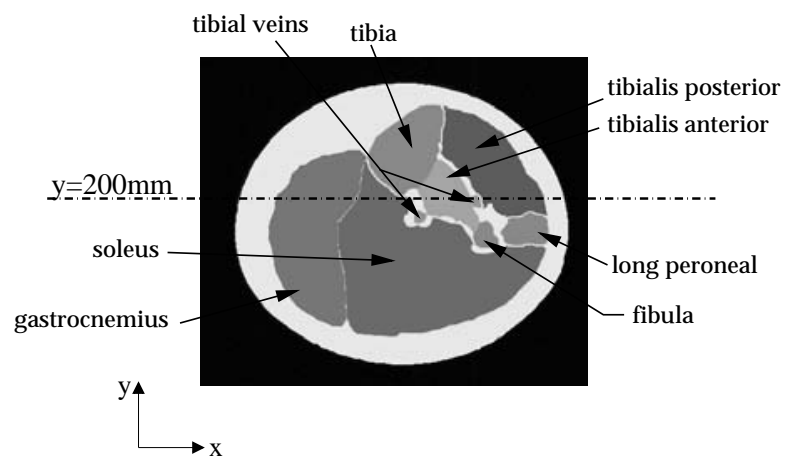

Figure 4. Calf geometry after segmenting process.

\subsection{Building the FE model}

The simulation is performed in order to obtain the calf response under the stocking compression. This 2D simulation is separated into two sequences. The first step 
consists in putting the sock in tension so that it will compress the calf when it will relax in the second step. The tension is obtained by applying a pressure inside the sock. It can also be added that the numerical model is formulated within the framework of nonlinear finite strains because of the use of hyperelasticity. The whole model has been considered hyperelastic, composed of 8 different materials. They are all chosen as a type of Mooney-Rivlin. The constitutive parameters used in the simulation are presented in the table 1 . These ones were chosen experimentally, based on models already existing in our code (Transvalor/ENSMP et al., 2006).

Table 1. Parameters used for the presented simulation.

\begin{tabular}{|c|c|c|c|c|}
\hline & $c_{10}$ & $c_{01}$ & $c_{20}$ & bulk modulus \\
\hline garment & 1. & 2. & -0.02 & 100 \\
\hline adipose & 1.4 & 1. & -0.04 & $10^{8}$ \\
\hline gastrocnemius & 0.9 & 0.6 & 0. & $10^{8}$ \\
\hline tibialis posterior & 1.1 & 0.6 & 0. & $10^{8}$ \\
\hline tibialis anterior & 1.2 & 0.7 & 0. & $10^{8}$ \\
\hline long peroneal & 1.1 & 0.7 & 0. & $10^{8}$ \\
\hline tibial veins & 1.3 & 0.4 & 0. & $10^{8}$ \\
\hline soleus & 1. & 0.6 & 0. & $10^{8}$ \\
\hline
\end{tabular}

As the data to be compared in the identification process are displacements, results here are displacements along the two principal axes on the whole section and then, on a cutting plane where the calf is the largest i.e. approximately $\mathrm{y}=200 \mathrm{~mm}$. It has been verified that these results follow the same trend as correlation results obtained previously. The first aim is to compare element order of integration, displacement results and time calculation. The first set of displacement results is obtained with linear under-integrated elements in $1326 \mathrm{~s}$ (CPU time) with a Pentium 4, 3.2GHz processor. The use of under-integrated elements is firstly chosen because of the mixed formulation. As it was presented before (Section 2.5) this type of mixed formulation is mandatory, but to keep the problem solvable the unknowns in pressure must be less numerous than the kinematical unknowns per element. It means that incompressibility constraints are less numerous than with standard linear element and the calculation time will decrease with the order of integration.

Considering the same simulation with fully-integrated and under-integrated quadratic elements, it gives an increase of only $9 \%$ of calculation time when using full-integrated ones. This simulation with linear under-integrated elements takes $1200 \mathrm{~s}$ which is 4.5 times less than with quadratic ones. The displacement distribution presents differences between results obtained with the two types of element. Actually, a ratio of 10 is observed on horizontal displacements (Figure 5) but the displacement changes follow similar trends. On the contrary, vertical displacements are of the same order of magnitude (Figure 6) but present large differences in their changes. Further- 


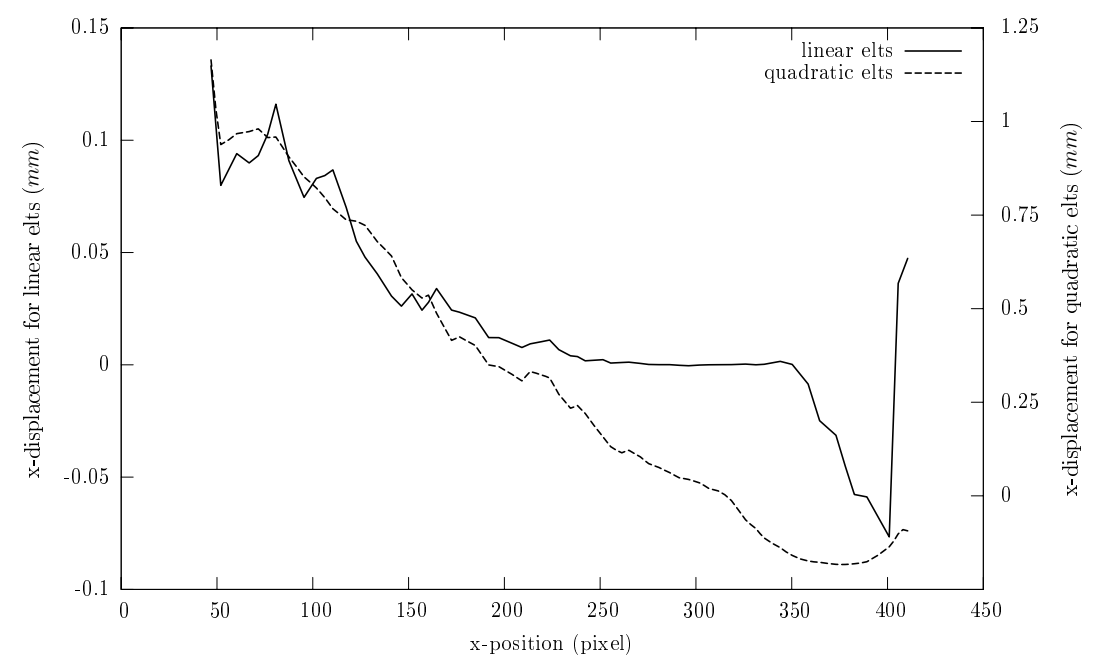

Figure 5. Horizontal displacements obtained with linear and quadratic underintegrated elements at $y=200 \mathrm{~mm}$.

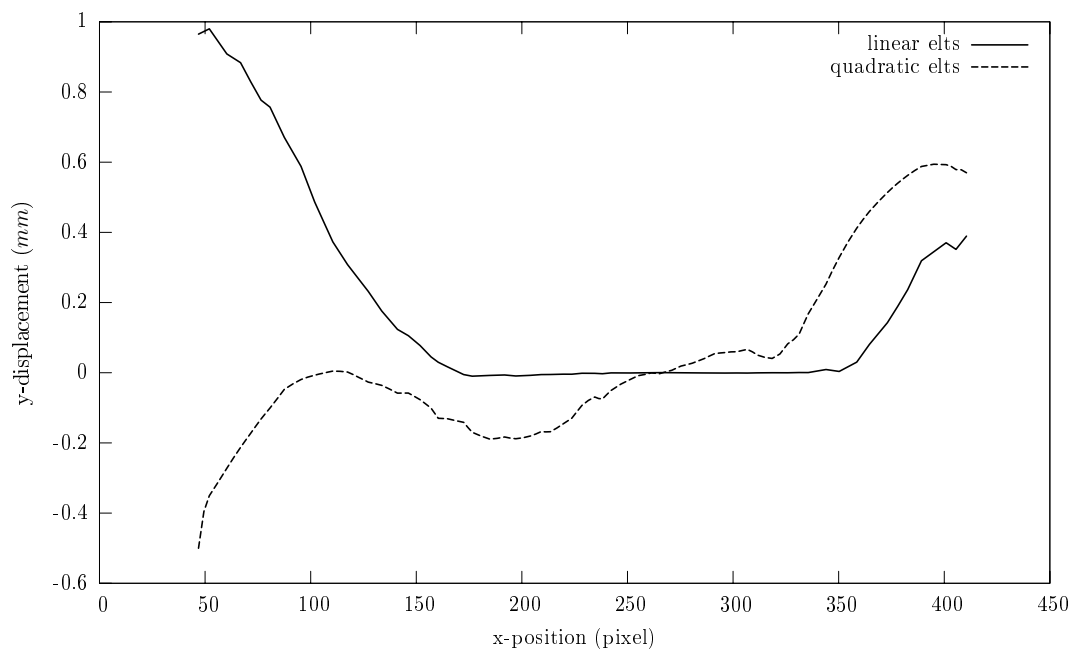

Figure 6. Vertical displacements obtained with linear and quadratic under-integrated elements at $y=200 \mathrm{~mm}$. 
more, if we compare vertical displacements (Figure 6) obtained with linear elements with those obtained with quadratic ones, we can observe that on the left side of the calf some values are positive in one case and negative in the second case. According to the experimental results (Figure 3), vertical displacements should be negative on the left side of the leg, this corresponds to the results obtained with quadratic elements.

On figure 7 , it can be observed that the pressure obtained with linear mixedelements is highly discontinuous. It is confirmed by the following figure (Figure 8), where the pressure plotted as a function of the position $x$ along the line at $y=200 \mathrm{~mm}$ (see Figure 4) oscillates from negative to positive values. Furthermore, a ratio of, at least, 50 is observed for the maximal pressure between linear and quadratic elements. This ratio may be due to the constant number of elements, involving variable number of DOF. In the linear case, 11297 DOF are used as 25514 are used in the quadratic case. But other cases tested, considering compressible materials, show that it is definitely the simultaneous treatment of both incompressibility and contact which gives rise to severe numerical difficulties for linear elements, while quadratic elements behave properly. To summarize, quadratic under-integrated elements will be used in our strongly nonlinear problem, so that pressure and displacements are better predicted.

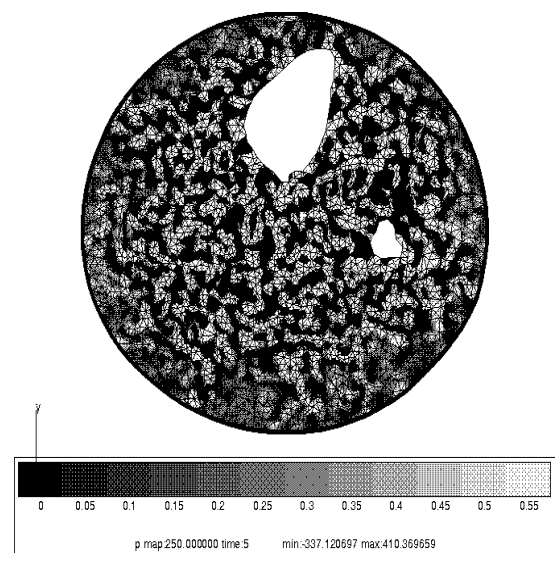

(a)

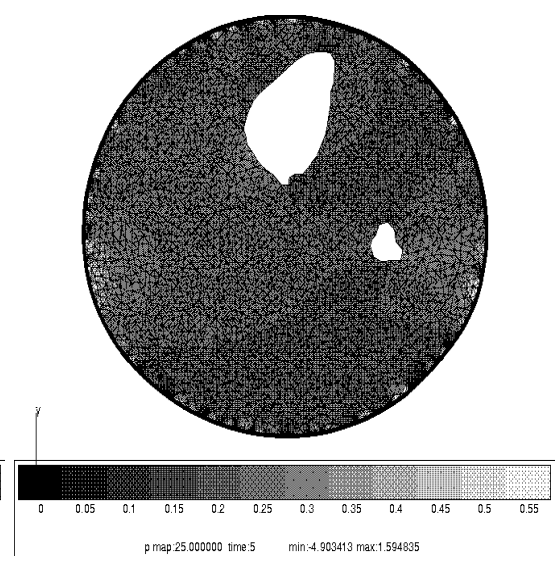

(b)

Figure 7. Pressure obtained with linear (a) and quadratic (b) elements.

\subsection{Identification results}

Before applying the identification process to the real data, a first test has been done. The idea is to compare two types of optimization algorithms. On the one hand, a simple simulation (a disk under radial loading) is performed and strains in some points are extracted which compose the experimental data. On the other hand the same simulation is performed but with some unknown material parameters. The aim 


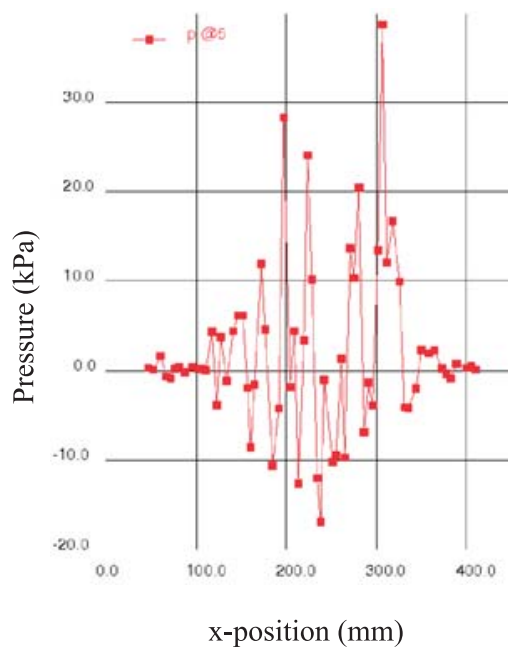

(a)

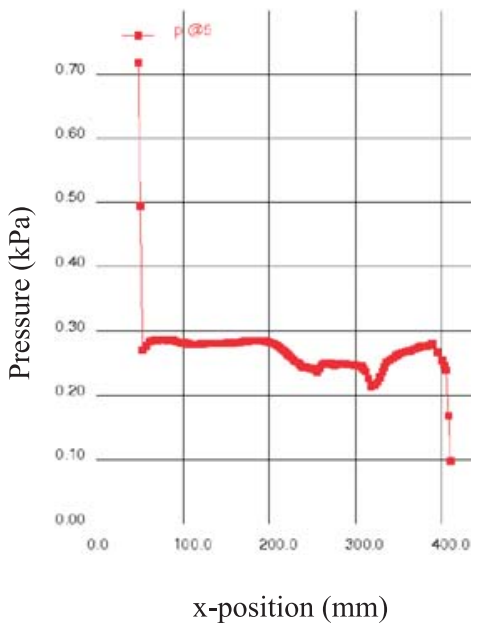

(b)

Figure 8. Pressure vs position along the line at $y=200 \mathrm{~mm}$ (see Figure 4), obtained with linear (a) and quadratic (b) elements.

is to determine which algorithm is the most efficient to identify the original material parameters. A linear elastic material and a two-term Mooney-Rivlin material are considered, corresponding to 3 parameters to be identified, namely, the Young modulus, $c_{10}$ and $c_{01}$ (see Section 2.4). For this case, the Levenberg-Marquardt (Rao, 1996) and the Nelder-Mead algorithm (Luersen et al., 2004) are compared. It is observed that the Nelder-Mead (NM) algorithm converges more slowly than the Levenberg-Marquardt (LM) one . With the same accuracy, for identifying two hyperelastic material parameters and an elastic one the NM algorithm takes 202 iterations (in 874 s) while the LM one takes 49 iterations (in $210 \mathrm{~s}$ ). This first result has to be confirmed in the future when it is applied to the model detailed previously.

\section{Conclusion}

This study coupling MR techniques and Finite Element Method to determine material parameters is motivated by the lack of information on the deep venous system and the need of an in vivo non-invasive methodology for an identification process of soft biological tissues. It has been explained in Section 3.1 that correlation windows could be used in different sizes and shapes. The choice made is justified by many observations. So, a way to assess the quality of our results is to modify the type of the windows used (shape, size...) but it could also be done by increasing their num- 
ber and by a different "distribution map". These changes will not have to modify our forthcoming identification results in order to consider correlation results satisfactory. The FEM requires a realistic mesh so we may have to use a large number of elements. Nevertheless, a less refined zone where no displacement is got experimentally can be considered, while a more refined zone around the vascular area is essential. In such a strongly nonlinear problem, the principal difficulties do not appear in the mesh definition but in the choice of the type of elements. This part of the study which consists in validating the numerical model must be achieved before making further comparison between experimental and simulated data. However, qualitative considerations can be made such as the displacement and pressure trends, and permitted to select the proper element type. The identification process has not been done while the computation is not fully validated. At the moment, we are studying the possibility to use another model which will permit to avoid solving contact problems, to get the first results of the identification process.

\section{References}

Bosboom E. M., Hesselnk M. K., Oomens C. W., Bouten C. V., Drost M. R., Baaijens F. P., «Passive transverse mechanical properties of skeletal muscle under in vivo compression », Journal of Biomechanics, vol. 34, $\mathrm{n}^{\circ}$ 10, p. 1365-1368, 2001.

Drapier S., Gaied I., « Identification strategy for orthotropic knitted elastomeric fabrics under large biaxial deformations », Inverse Problems in Science and Engineering, vol. 15, p. 871894, 2007.

Ducottet C., Daniere J., Moine M., Schon J. P., Courbon M., « Localization of objects with circular symmetry in a noisy image using wavelet transforms and adapted correlation », Pattern Recognition, vol. 27, p. 351-364, 1994.

Fung Y. C., Biomechanics: mechanical properties of living tissues, Springer, Berlin, 1993.

Gaied I., Drapier S., Lun B., « Experimental assessment and analytical 2D predictions of the stocking pressures induced on a model leg by Medical Compressive Stockings », Journal of Biomechanics, vol. 39, p. 3017-3025, 2006.

Luersen M. A., LeRiche R., « Globalized Nelder-Mead method for engineering optimization », Computers and Structures, vol. 82, $\mathrm{n}^{\circ}$ 23-26, p. 2251-2260, 2004.

Ogden R. W., « Elastic deformations of rubberlike solids », in , H. Hopkins, , M. Sewell (eds), Mechanics of solids : the Rodney Hill 60th anniversary volume, Pergamon Press, p. 499537, 1982.

Rao S. S., Engineering optimization[Texte imprimé]: theory and practice, Wiley, New York, 1996.

Sussman T., Bathe K.-J., «A finite element formulation for nonlinear incompressible elastic and inelastic analysis », Computers and Structures, vol. 26, n 1-2, p. 357-409, 1987.

Transvalor/ENSMP, Northwest Numerics I., Z-mat Version 8.3.6. 2006.

Weiss J. A., A constitutive model and finite element representation for transversely isotropic soft tissues, PhD thesis, Dept. of Bioengineering, University of Utah, 1994. 